\title{
A Clinical Decision Support System Design Framework for Nursing Practice
}

\author{
Sheng-Chieh Lu ${ }^{1}$ Rebecca J. Brown ${ }^{2}$ Martin Michalowski \\ ${ }^{1}$ Department of Symptom Research, University of Texas MD Anderson \\ Cancer Center, Houston, Texas, United States \\ 2 School of Nursing, University of Minnesota, Minneapolis, Minnesota, \\ United States \\ Address for correspondence Sheng-Chieh Lu, PhD, Department of \\ Symptom Research, University of Texas MD Anderson Cancer Center, \\ 6565 MD Anderson Boleuvard, Houston, TX 77030, United States \\ (e-mail: slu4@mdanderson.org).
}

ACI Open 2021;5:e84-e93.

Abstract

Keywords
- clinical decision
support
- evidence-based
practice
- knowledge repository
- analysis

Background As nurses increasingly engage in decision-making for patients, a unique opportunity exists to translate research into practice using clinical decision support systems (CDSSs). While research has shown that CDSS has led to improvements in patient outcomes and nursing workflow, the success rate of CDSS implementation in nursing is low. Further, the majority of CDSS for nursing are not designed to support the care of patients with comorbidity.

Objectives The aim of the study is to conceptualize an evidence-based CDSS supporting complex patient care for nursing.

Methods We conceptualized the CDSS through extracting scientific findings of CDSS design and development. To describe the CDSS, we developed a conceptual framework comprising the key components of the CDSS and the relationships between the components. We instantiated the framework in the context of a hypothetical clinical case.

Results We present the conceptualized CDSS with a framework comprising six interrelated components and demonstrate how each component is implemented via a hypothetical clinical case.

Conclusion The proposed framework provides a common architecture for CDSS development and bridges CDSS research findings and development. Next research steps include (1) working with clinical nurses to identify their knowledge resources for a particular disease to better articulate the knowledge base needed by a CDSS, (2) develop and deploy a CDSS in practice using the framework, and (3) evaluate the CDSS in the context of nursing care.

\section{Background and Significance}

Clinical decision support systems (CDSSs) are linked to improvements in preventive services, ordering of clinical tests, medication prescription, and financial outcomes. ${ }^{1-3}$ As nurses increasingly engage in decision-making for patients, utilization of CDSSs to support nursing becomes

received

December 10, 2020

accepted after revision

September 2, 2021
DOI https://doi.org/

$10.1055 / \mathrm{s}-0041-1736470$.

ISSN 2566-9346. more prevalent internationally. ${ }^{4-6}$ There is a unique opportunity in nursing to translate research into practice using CDSSs because nursing interventions are often (a) inexpensive and (b) can be implemented with less administrative burden as nursing interventions are not linked to a prescription, international classification of diseases codes (ICD), or current procedural terminology code. ${ }^{7,8}$

\section{(C) 2021. The Author(s).}

This is an open access article published by Thieme under the terms of the Creative Commons Attribution License, permitting unrestricted use, distribution, and reproduction so long as the original work is properly cited. (https://creativecommons.org/licenses/by/4.0/)

Georg Thieme Verlag KG, Rüdigerstraße 14, 70469 Stuttgart, Germany 
Clinical decision support applications have been applied to various nursing tasks, including patient assessment and care planning for fall, pressure ulcer, pain, blood glucose level, triage, and patient referral. ${ }^{4,9}$ Research has shown that CDSS leads to improvements in patient outcomes and nursing workflow, ${ }^{4,9,10}$ yet the success rate of CDSS implementation in nursing is low. ${ }^{10-12}$ Multiple complex factors contributing to limited adoption of CDSS have been documented, such as the lack of integration of workflow and CDSS, and the consideration of organizational settings and cultures. ${ }^{13}$ Another notable barrier to implementation is lack of CDSSs that have a capability to support complex patient care $^{4,9}$

\section{Nursing Decision-Making Process}

Several models have been applied to describe and understand nursing decision-making processes. ${ }^{14,15}$ An emerging model is the dual-process theory described in the cognitive psychology literature and increasingly discussed in nursing literature. ${ }^{16}$ The theory describes that humans use two separate reasoning systems, system 1 (S1) and 2 (S2), to make decisions. The $\mathrm{S} 1$ is a fast and automatic form of judgments based on intuitive, tacit knowledge, and experience, while the $\mathrm{S} 2$ reasoning is a slow, rule-based, analytical form of judgments that requires additional information. ${ }^{17}$ Literature has shown that nurses use both forms of reasoning in their practice of decision-making. ${ }^{18}$ The use of experience and tacit knowledge to recognize the similarities between patient patterns and makes decisions according can be characterized as S1. 6,17,19 Research has indicated that this way of knowing enables quick decisions with comparable accuracy in certain situations. ${ }^{20}$ However, a body of literature argues that decisions made using S1 are subject to cognitive biases, such as stereotyping biases, anchoring, and confirmation biases, ${ }^{21,22}$ and may be suboptimal.

On the other hand, nurses adopt the $\mathrm{S} 2$ process to make decisions for less familiar situations. ${ }^{14} \mathrm{~S} 2$ emphasizes the use of a logical process to determine actions in addressing patient needs according to the combination of patient cues and scientific evidence. The use of the $\mathrm{S} 2$ in decision-making increases the communicability of nursing decision-making, allows decision replication and reevaluation, and reduces the probability of suboptimal decisions. ${ }^{14}$ However, adopting S2 process is challenging in real-world nursing practice due to the lack of information accessibility and limited time for decision-making. ${ }^{14,23}$ Further, it becomes challenging when making decisions for complex situations, such as selecting interventions for patients with comorbidities.

Although there is an ongoing debate about whether S1 and S2 are equal partners or S2 functions to correct biased decisions from $S 1,{ }^{17,19,24}$ most studies concluded that there is a need to find a way to incorporate scientific evidence with nursing experience in making decisions. ${ }^{17,25,26}$ Adopting a CDSS addresses the time and information restrictions and promotes the use of evidence in nursing decision-making. ${ }^{27}$ Thus, we performed a study, currently under review, that categorizes the type of learning done by nurses of varying experience levels, and we sought to conceptualize a frame- work that incorporates the findings of that study for future CDSS development in this study.

\section{The CDS Five Rights Framework}

The CDS Five Rights framework, proposed by Osheroff and colleagues, guides the development and implementation of CDSSs that provide the right information to the right person through the right format and channel at the right time. ${ }^{28}$ The right information refers to communicating context-specific, evidence-based, and actionable information to clinicians. The information needs of nurses vary with tasks supported by a CDSS. ${ }^{29}$ Providing information that is relevant to the task at hand is key to effective decision support. ${ }^{30}$ Different tasks involve different decisions and thus require different information and knowledge to support these decisions. Identifying and understanding the tasks to support are essential steps for CDSS development. Research shows that information delivered by a CDSS driven by evidence that is congruent with users' core values and organization policies increases its usefulness and acceptability. ${ }^{28}$

Scientific efforts have been made to understand nurses' information-seeking behaviors and common information resources. The resources include clinical practice guidelines (CPGs), textbooks, organizational protocols, clinical publications, and the insights of clinical experts and colleagues. ${ }^{31-33}$ Nurses especially value the knowledge they learned from their past experience, and studies consistently reported that the first source of information for nurses is their colleagues. ${ }^{29}$ Colleagues include fellow nurses, nursing managers, clinical nurse specialists, and other health care professionals. $^{32}$ Through merging the opinions from colleges and experts using consensus, one can move this anecdotal approach toward evidence basis such as that achieved through CPG. ${ }^{34}$

Communicating information to individuals in need refers to the right person. ${ }^{28}$ Nurses play divergent roles in patient care and have varied decision support needs. ${ }^{35}$ For instance, bedside nurses need detailed information about treatments for patient care, while rapid response team nurses need information to support their decision on whether a patient needs a higher level of care. ${ }^{36}$ Research also recommends early CDSS end-user involvement in CDSS development to enhance its implementation and adoption. ${ }^{30,37}$ The end users provide key insights of improving CDSS usefulness and serve as the early adopters to promote the implementation. ${ }^{12}$ Identifying target users and engaging them is required to develop an effective CDSS.

The right format describes the types and formats of decision support interventions appropriate and preferred by CDSS end users. ${ }^{10,28}$ A taxonomy for decision support interventions was previously described to guide CDSS development. $^{28}$ The taxonomy includes five categories and ten different formats of decision support for a variety of clinical tasks ( - Table 1 ). In addition, a previous study revealed that novice nurses preferred detailed information, while experienced nurses preferred brief messages with high relevancy. ${ }^{38}$ This finding indicated that the best CDSS interfaces vary across users with different backgrounds and features. 
Table 1 Formats of decision support interventions

\begin{tabular}{|c|c|}
\hline Decision support interventions & Description \\
\hline \multicolumn{2}{|l|}{ During data-entry tasks } \\
\hline Smart documentation forms & $\begin{array}{l}\text { - Present documentation items to enhance completeness and correctness of } \\
\text { documentation for care quality, legal requirements, and reimbursement }\end{array}$ \\
\hline $\begin{array}{l}\text { Order sets, care plans, and } \\
\text { protocols }\end{array}$ & $\begin{array}{l}\text { - Provide predefined order sets or care plans based on care policies, standard guidelines, } \\
\text { and local protocols to enhance adherence to best practices }\end{array}$ \\
\hline Parameter guidance & $\begin{array}{l}\text { - Provide suggested dosage and frequencies of drugs and interventions based on a } \\
\text { predefined algorithm and other evidence during prescribing }\end{array}$ \\
\hline $\begin{array}{l}\text { Critiques and warnings } \\
\text { (immediate alerts) }\end{array}$ & $\begin{array}{l}\text { - Provide alerts regarding potential errors, hazards, and quality improvement } \\
\text { opportunities related to new patient data and interventions entered }\end{array}$ \\
\hline \multicolumn{2}{|l|}{ During data-review tasks } \\
\hline $\begin{array}{l}\text { Relevant data summaries } \\
\text { (single patient) }\end{array}$ & - Provide concise summary data specific to a patient for planning and evaluating care \\
\hline Multi-patient monitors & $\begin{array}{l}\text { - Provide organized key data, parameters, and information covering several patients for } \\
\text { decisions on prioritizing patient care and resource distribution }\end{array}$ \\
\hline $\begin{array}{l}\text { Predictive and retrospective } \\
\text { analytics }\end{array}$ & $\begin{array}{l}\text { - Provide patient-specific suggestions generated by automatic algorithms based on } \\
\text { patient data }\end{array}$ \\
\hline \multicolumn{2}{|l|}{$\begin{array}{l}\text { During assessment and } \\
\text { understanding tasks }\end{array}$} \\
\hline $\begin{array}{l}\text { Filtered reference information } \\
\text { and knowledge resources }\end{array}$ & $\begin{array}{l}\text { - Provide up-to-date, filtered, and organized information and knowledge through info } \\
\text { buttons or links }\end{array}$ \\
\hline $\begin{array}{l}\text { Expert work-up and } \\
\text { management advisors }\end{array}$ & $\begin{array}{l}\text { - Provide suggestions, including diagnoses, tests, assessments, and interventions, based } \\
\text { on entered patient data }\end{array}$ \\
\hline \multicolumn{2}{|l|}{ Not triggered by a user task } \\
\hline $\begin{array}{l}\text { Event-driven alerts and } \\
\text { reminders }\end{array}$ & $\begin{array}{l}\text { - Provide notifications regarding new test results and special events that are not part of } \\
\text { routine practice }\end{array}$ \\
\hline
\end{tabular}

Source: Adapted and summarized from Osheroff and colleague. ${ }^{28}$

The right channel defines the delivery vehicle for a CDSS. A CDSS should deliver information at the place that users can perceive and use the information. ${ }^{28}$ For example, an admission assessment module might be an ideal place for nursing diagnosis suggestions. Another consideration for choosing where to embed a CDSS is data triggering the CDSS. Most CDSSs supporting nursing practice need patient data entered by nurses to initiate their functions. ${ }^{4}$ Providing decision support during patient data entry will enhance CDSSs' usability, usefulness, and acceptability. In addition, the vehicle for delivering information should show the information in a preferred format. Text-based information is appropriate to be delivered in a module of an EHR, while patient condition alerts need a mobile device to ensure user notifications are timely.

The right time refers to appropriate timing for delivering decision support. ${ }^{28}$ Providing information too frequently or at an incorrect time is a major barrier to implementing CDSSs in nursing. ${ }^{10}$ Providing information at an inappropriate time disrupts nurse's workflow and results in mistakes. Another well-documented barrier is alarm fatigue caused by frequent information. ${ }^{39}$ A decision support message delivered at the moment that target audiences are unable to receive it or take actions is oftentimes dismissed and decreases users' trust. Over time, users may develop alarm fatigue and treat all messages from CDSSs as insignificant. In such a case, the effectiveness of CDSS is reduced or even negative. ${ }^{39}$ Thus, providing the right information is not enough for decision support if the information is provided at the wrong moment.

The usefulness of the CDS Five Rights framework in developing effective decision support interventions for physicians has been previously discussed. ${ }^{40,41}$ However, the potential of the framework for guiding CDSS development for nursing practice has not yet been examined. In addition, although the CDS Five Rights framework highlights the essentialness of the correct information content and representation for a CDSS to be effective, the framework provides less guidance on how the information should be generated.

\section{Reasoning-Based Clinical Decision Support Systems}

As patient complexity increases, nurses are typically faced with multiple treatment options from several guidelines and protocols, increasing the likelihood of conflicts and causing adverse reactions. ${ }^{42}$ The evidence synthesis process is challenging for inexperienced nurses as there are many restrictions including lack of time, information resources, and knowledge to comprehend scientific articles. ${ }^{43,44}$ Senior nurses may also be overwhelmed by the number of options and conflicts between evidence, especially when they encounter unfamiliar patient conditions. Previous research has addressed this issue using reasoning-based CDSSs. ${ }^{45,46}$ One 
example is the work of Wilk et $\mathrm{al}^{46}$ that proposed a mitigation CDSS framework using a reasoning-based algorithm to generate optimized and conflict-free suggestions from multiple CPGs for supporting multimorbidity patient care at the point-of-care. A similar optimizing process can be used to eliminate duplicative and conflicting nursing interventions, sequencing the interventions, and suggesting frequencies of the interventions from multiple evidence. For instance, a CDSS providing summarized content of patient education regarding nutrition and physical activity based on multiple guidelines for diabetes and hypertension would reduce nurses' burden and enhance patient education quality. However, the previous work focused on mitigating conflicts between drugs. The same concept has not yet been applied to nursing practice. Furthermore, the mitigation CDSS framework is not concerned with the delivery of decision support messages to maximize their impact.

\section{Objectives}

Our goal is to develop a CDSS with a solid theoretical foundation for supporting complex patient care for nurses of varying experience levels to enhance care quality and patient outcomes. To achieve the goal, we first conceptualize the essential components that a CDSS for the practice should include based on literature and nursing decision-making theory. Specifically, we expand the CDS Five Rights framework by adding two components related to information generation to draw a conceptual framework depicting the CDSS.

\section{Methods}

To conceptualize the CDSS supporting complex patient care for nursing, we extracted insights for scientific findings of CDSS design and development from literature in several areas, including nursing information-seeking behaviors, nursing decision-making, and nursing clinical decision-making support system. Specifically, we worked with a senior university librarian to identify relevant literature from Ovid Medline. We searched the following concepts using subject headings and keywords as needed: "decision making," "nursing," "evidence-based practice," "electronic health records," "decision support systems," and "workflow." We included all literature discussing clinical information-seeking behavior, decision-making process, decision-making theory, and decision support system design and evaluation.

A graduate-level research assistant then abstracted and summarized the insights from the literature for conceptualizing the CDSS and developed a conceptual framework to depict the key components of the CDSS and relationships between the components. We present the summarized insights in the background section. The conceptualization process was completed through iterative discussions among our team members consisting of a senior researcher in artificial intelligence, an experienced nursing informatician, and an experienced registered nurse. Finally, we utilized a hypothetical clinical case developed by a cardiovascular and pulmonary nurse to demonstrate the development of the CDSS using the framework.

\section{Results}

Our initial conceptual framework for the CDSS supporting complex patient care for nursing practice was largely overlapped with the CDS Five Rights framework. Therefore, we decided to inherit the CDS Five Rights framework and expand it. As a result, we proposed six interrelated components for conceptualizing the CDSS (-Fig. 1). Of the six components, four correspond to the CDS Five Rights framework. Instead of using original terminology, we chose to use "Who," "What," "When," and "User interface" to make the components more straightforward. The two unique components, knowledge repository and reasoning system, are included to explicitly highlight how an evidence-based CDSS generates optimized decision support messages. A reasoning system is a system that has the capability to synthesize available knowledge and environmental information to drive conclusions or predictions using a set of predefined rules, ${ }^{47}$ while a knowledge repository stores various clinical knowledge in a computerreadable format to support the reasoning system. ${ }^{48}$ In the following subsections, we provide descriptions of each component in the context of a hypothetical clinical case describing care for patients with congestive heart failure (CHF) (-Table 2). We provide the description of each bolded component in - Fig. 1 in its corresponding subsection below.

\section{Who}

The "Who" component refers to the target end users of a CDSS. As discussed previously, end users have many implications for CDSS development. The end users are knowledgeable about what, when, where, and how their tasks need to be supported by a CDSS, and thus early end-user identification and engagement enhance the usefulness and adoption of the CDSS.

In the hypothetical clinical case, the primary end users for the CDSS are nurses working on a step-down telemetry unit caring for CHF patients. Engaging Nurse Y as a representative of target end users to ensure a context-specific and userpreferred CDSS is needed during CDSS development. The representative provides insights from the end users' perspective and helps guide decision-making on CDSS design concerning the rest of the components.

\section{What}

The ability to deliver the right information requires understanding what tasks to support and what corresponding information supports the task. A thorough understanding of the tasks is essential to determine what decisions are involved, what information is required to inform the decisions, and what knowledge should be stored in the knowledge repository. Several task analysis methods have been developed ${ }^{49,50}$ to identify and analyze the target tasks through collaboration with the end users once they are engaged.

The primary task in caring for Mrs. Thompson is choosing the optimal nursing diagnosis and evidence-based 


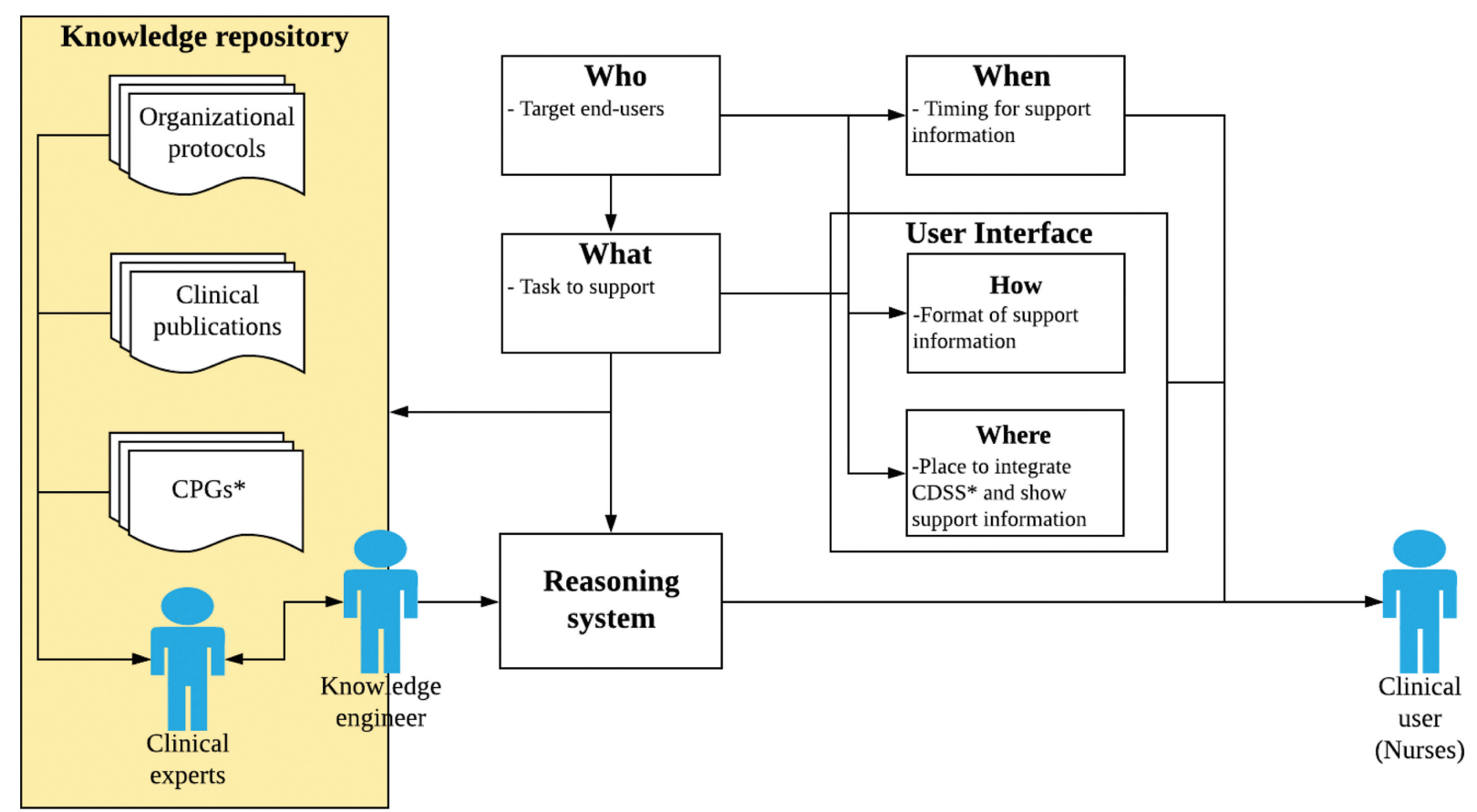

Fig. 1 Framework for developing clinical decision support systems to support nursing. Abbreviations: CDSS, clinical decision support system; CPGs, clinical practice guidelines.

Table 2 Clinical case for illustrating our CDSS framework

Nurse Y's patient, Ms. Thompson, is a 75-year-old Black female and has been admitted to Hospital X for the second time this month for heart failure exacerbation. She is short of breath, with crackles in her lungs and requiring two liters of oxygen via nasal cannula. She also reports having persistent chest pain for the past 3 months that does not seem to get much better or worse. Her oxygen saturation level is $92 \%$ on room air and her respiratory rate is fast at 28 breaths per minute. Her heart rate is 104 beat per minute, in sinus tachycardia. Her blood pressure is 138/62. She is afebrile. She reports having to sleep upright in her recliner at home and is exhausted from not being able to sleep comfortably. Her weight is up seven pounds since last week. She is oriented to person, place, and time though she is sometimes confused about why she is in the hospital. At times she expresses she is anxious and fearful about being in the hospital again and she is constipated. She has a history of left and right heart failure with reduced ejection fraction, coronary artery disease, diabetes mellitus Type II (last Hgb A1C 6.8\% and taking metformin), hypertension (taking verapamil), and dyslipidemia. She had a non-ST segment elevation myocardial infarction 12 years ago requiring percutaneous coronary intervention. Patients with CHF may battle with shortness of breath, fatigue, reduced cardiac output, fluid overload, inadequate tissue perfusion, and exercise intolerance. There are multiple nursing therapies for each attribute of this clinical syndrome. Nurses prioritize diagnoses and interventions to target each shift. To support nursing care for patients with similar conditions with Mr. Thompson, Hospital X decides to develop a new CDSS supporting the care. A CDS development team is convened to develop the CDSS, and the team includes two senior engineers, a nursing informatician, and Nurse $Y$ as representative of target users. The goal of the development team is to develop a CDSS helping nurses toggle through and select an appropriate care plan for their patients with CHF and other comorbidities.

interventions, which can be well supported by a CDSS. The development team then observes and interviews several nurses in the step-down telemetry to determine what decisions are involved in nursing diagnosing and intervention planning for congestive heart failure patients.

\section{Reasoning System}

Research suggests applying evidence-based reasoning processes to make decisions that can be validated and communicated. ${ }^{6}$ As the complexity of patient conditions and evidence increases, nurses need support in their evidencebased decision-making. Adopting a reasoning-based algorithm in a CDSS has been proven useful in generating actionable, prioritized, and conflict-free recommendations by providing synthesized-evidence from multiple resources and patient-specific decision support at the point of care. ${ }^{46}$ In addition, a reasoning system could help standardize the "art" of nursing to better reflect the gains achievable when merged with the "science" of nursing.

In this use case, several clinical guidelines, protocols, and evidence are applied to heart failure patients due to the disease's complexity and comorbidities and can result in conflicts and duplicates. For example, physical activity recommendations vary across the guidelines for hypertension, diabetes, and heart failure. ${ }^{51-53}$ Nurse Y needs to consider all applicable guidelines and summarize them to provide Ms. Thompson with individualized and actionable education on physical activity. A CDSS providing knowledge from all guidelines does not work well in this situation. There must be reasoning in the CDSS to prioritize the patient's problems 
and synthesize all evidence, providing optimized patientspecific suggestions to address the adverse interactions and support the nursing decision-making for determining the content of patient education for Ms. Thompson.

\section{Knowledge Repository}

A reasoning system requires a knowledge repository containing preprocessed knowledge to function. Knowledge in a repository should be up-to-date and from the user's preferred sources. ${ }^{28}$ We explicitly include several commonly reported knowledge resources in nursing to guide a knowledge repository development. The resources include organizational protocols, journal publications, and CPGs. ${ }^{32}$ Working with clinical experts is essential as their experiences provide additional knowledge specific to their practice and confirm the relevancy and usefulness of the included evidence to enable a thorough repository. After collecting knowledge from all available resources, it is necessary to include a knowledge engineer to translate the knowledge into a computer-interpretable format.

The development team works on identifying knowledge resources applicable to Mrs. Thompson's care to construct the knowledge repository. Knowledge resources are identified, including: (1) the NANDA International Nursing Diagnosis textbook, ${ }^{54}(2)$ medical, surgical, and cardiovascular textbooks, (3) best-practice guidelines from professional organizations, (4) clinical reference tools such as DynaMed, and (5) input from clinicians and professionals such as cardiologists, advanced practice registered nurses, physician assistants, pharmacists, registered nurses, and cardiovascular researchers. ${ }^{55}$

\section{When}

The "When" component refers to appropriate timing in workflow when decision support is used. Providing decision support at inappropriate moments would be ineffective, distracting, and considered a nuisance. The best timing for supporting nursing decisions varies by tasks and individuals. Individuals have differing preferences regarding the timing for showing the decision support intervention even if they shared the same task. ${ }^{30}$ However, literature on appropriate timing is severely limited due to variations between settings and institutions for specific tasks. It is suggested to work with the end users to find out the optimal timing and allow individuals to customize the timing to match their workflow. ${ }^{30}$

For this clinical use case, several timings are appropriate for providing decision support. To accommodate different preferences nurses may have, the development team builds the CDSS to allow each nurse to customize the timing for showing recommendations. Nurses can choose to receive recommendations whenever they enter particular patient data or after they complete the entire assessment in a flowsheet through indicating their preferences in personal profiles.

\section{User Interface}

The "User interface" component covers how a CDSS interacts with end users. There are two subcomponents, "How" and "Where."

\section{How}

The "How" component refers to the appropriate and preferred decision support format and is related to the right format. Decision support can be delivered in any number of ways, and the correct delivery greatly contributes to the effectiveness of a CDSS. The ideal format for a specific task, again, is task and target population varied. Numerous formats to deliver decision support were developed, and the best ways to utilize each format were described by Osheroff et al. ${ }^{28}$ ( - Table 1). A thorough understanding of each format is essential for CDSS developers. Besides, the characteristics of end users have influences on optimal decision support delivery. ${ }^{9,38}$

To achieve the goal set for the CDSS in the clinical use case, "Expert work-up and management advisors" (-Table 1) is suitable. The task of the CDSS supporting the care of Mrs. Thompson is to provide conflict-free and prioritized interventions according to diabetes, hypertension, and heart failure guidelines. To minimize interruptions but to clearly alert the nurse to the support message, the development team designs a module to present the prioritized list of interventions and makes the module available in the amble bar of Mrs. Thompson's screen. This format is preferred to a pop-up window disrupting nurse workflow, particularly for experienced nurses. New nurses, however, can still easily access this information and incorporate it into their practice.

\section{Where}

The "Where" component, corresponding to the right chan$n e l$, refers to the location for CDSS implementation. The primary concern is whether end users can easily see and use the decision support. Furthermore, most CDSSs require patient data entered by nurses or accessed from other systems to function. ${ }^{4}$ Thus, where to get the data is another concern when deciding the vehicle for embedding the CDSSs.

Electronic health records are common vehicles for CDSSs since they are ubiquitous across most health care systems. ${ }^{56}$ Many EHRs have the capability to embed a CDSS into a particular module, such as a nursing flowsheet. Nursing flowsheets are spreadsheet-like and evidence-based templates for structurally documenting assessments, interventions, and other types of patient data. ${ }^{57}$ The structured nursing flowsheets are exceptional data sources for CDSS and places to incorporate it. ${ }^{57}$ Incorporating the CDSSs in nursing flowsheets that contain the data triggering the systems can efficiently reuse patient data and enhance the usability and usefulness of the CDSSs.

Embedding the CDSS in the EHR has several advantages. First, the CDSS can access patient data to generate decision support without extra data entry and display messages in text format. In the clinical use case, Nurse Y has amassed demographic, vitals, symptoms, and situational data that are all needed to develop a care plan and deliver therapies. Within the EHR, the CDSS can easily link these variable keys from multiple modules in the background and save nurses from re-entering data. 


\section{Discussion}

Clinical decision support studies in nursing have lagged behind compared with other health care professionals such as medicine. ${ }^{6}$ In this work, we conceptualized the CDSSs supporting decision-making during complex patient care for nursing and proposed a conceptual framework comprising six key components for depicting such a CDSS via expanding the CDS Five Rights framework. Specifically, we included two additional components, knowledge repository and reasoning system, to explicitly highlight the need and process of optimized decision support message generation for the CDSSs. As a result, our conceptual framework covers components for generating evidence-based, optimized decision support and presenting the decision support to end users. Through the hypothetical CHF case, we demonstrated that our conceptual framework can serve as a comprehensive roadmap guiding CDSS development and bridge scientific research and practice. Further, the conceptual framework is designed to be disease-agnostic through its use of general concept definitions. We use the hypothetical CHF case as an instance of the framework to demonstrate its applicability to a specific disease, and we believe the framework is generalizable to care of other diseases.

The inclusion of the reasoning system and knowledge repository components distinguishes our framework and highlights decision support generation of the CDSS. ${ }^{28,58}$ As evidence-informed clinical decision-making becomes a national priority, health care providers need applications that integrate evidence, such as CPGs, to support their decisions. Lack of time and accessibility of evidence are major barriers to evidence-based practice in nursing. ${ }^{59}$ Reasoning systems delivering prepackaged, patient-specific, and conflict-free decision support based on multiple evidence have shown promise to address the barriers and enhance clinical evidence adherence and improve patient outcomes. ${ }^{9,46,60,61}$ For instance, a CDSS with a reasoning system should have the capability to synthesize CHF and diabetes nursing care guide to suggest prioritized and duplicate-free nursing interventions for supporting nurses' care planning.

The knowledge stored in the knowledge repository influences the extent to which a reasoning system provides meaningful and actionable recommendations to decisionmakers. ${ }^{62}$ Research suggests that knowledge in a CDSS should come from the sources that match end users' beliefs and preferences. ${ }^{28,38}$ In our conceptual component, we list several evidence resources that are commonly used by nurses during decision-making. Of the included resources, clinical experts are included to verify and fructify the knowledge using their experience as nurses valued their colleagues' and administrators' knowledge when they encounter a decision-making point. ${ }^{31,32}$ Other knowledge resources, such as textbooks, can also be used to construct the knowledge repository, and the resources we included provide a general direction to work with end users to identify the best resources for the repository. In addition, we include a knowledge engineer as an interface between the knowledge repository and reasoning system to emphasize the need of knowledge translation and maintenance. Knowledge management keeping evidence in the repository up to date and high-quality requires additional considerations and is out of the scope of this study. However, we anticipate that the maintenance effort will be low given that updating a CPG or protocol typically requires several years. ${ }^{63}$ Further, some of the management could be automated using advanced artificial intelligence techniques in knowledge representation. ${ }^{62}$ With regard to knowledge quality control, we highlight the need to work with clinical experts to ensure that the best knowledge is included in our framework. As future work, we will study how to incorporate a robust knowledge management model into the framework.

The rest of the components underscore the aspects of decision support presentation to maximize the likelihood of successful adoption. We proposed these components based on what we learned from relevant literature, and high correlations between these components and CDS Five Rights indicate the importance of these components for a CDSS. Through the hypothetical clinical use case, we showed the use of the components to aggregate scientific findings of CDSS design to support CDSS development for CHF patients with comorbidity. During the aggregation, we noticed serval gaps in CDSS design. There were limited suggestions for some components, including "who," "what," and "when," as suggestions from studies may not always be applicable due to the variation in nursing practice and organizational regulations. Research is needed to identify methods to determine what, when, where, and how to deliver decision support interventions to address barriers to CDSS adoption, such as workflow interruption and organizational issues..$^{13,30}$

In the conceptual framework, all components emphasize the need to involve end-user representatives at an early stage in CDSS development, consistent with the notion that considering nursing as part of CDSS design is key to successful CDSS development and implementation. ${ }^{64}$ Literature shows that end-user involvement leads to CDSSs with better system quality, user satisfaction, and organization impact. ${ }^{65,66}$ In demonstrating our framework, we provide an exemplar of utilizing each component to work with the representative and use insights the representative provides. Additional prospective research is needed to examine whether such a user-centered framework leads to CDSSs that promote optimal nursing decisions and improve patient outcomes.

This study has limitations. First, our conceptual framework is not based on a systematic review. Our initial idea was to identify key components and insights of CDSSs to support our project developing and evaluating a CDSS supporting complex patient care for nurses. Second, our framework design was literature-driven and has not yet been evaluated with empirical research data. In addition, we conceptualized the framework mainly using nursing literature. Incorporating literature from other areas, such as medicine, can bring additional insights. Despite these limitations, the current results are promising as our findings on CDSS design are consistent among the literature we reviewed. Further discussions and trials evaluating the framework are needed to refine and valid it. 


\section{Conclusion}

We conceptualized the CDSS for complex patient care for nursing based on findings and theories in literature and proposed a conceptual framework describing the CDSS by expanding the CDS Five Rights framework. The conceptual framework aims to maximize the likelihood of a successful CDSS for the practice from a variety of perspectives into allencompassing design guidelines. Through the hypothetical use case, we demonstrate that the framework can bridge research findings in design and decision-making for CDSS development. This study highlights several areas for further investigation. First, inclusion of an end-user representative with knowledge of the task to support is key to effective CDSSs. However, an optimal way of selecting and involving an informative representative is still unclear. Second, studies have identified common information resources used to inform nursing decisions. ${ }^{29,32}$ However, the actual guideline clearinghouses and websites used in nurses' daily work are many and need to be better categorized. Finally, whether the framework presented in this paper leads to an effective CDSS to support nursing practice is worthy of further investigation. To address these issues, we conducted a survey with clinical nurses to understand their knowledge resource use to inform the knowledge base needed for a CDSS. We will incorporate the survey results to develop and deploy a CDSS using the proposed framework. Furthermore, our framework is significantly informed by literature from nursing research which pays less attention to CDSS development and evaluation than other disciplines. Incorporating the suggestions from literature focusing on other health care professionals, such as studies of CDSS for supporting physicians, will further refine our framework and enhance its applicability.

\section{Clinical Relevance Statement}

Effective clinical decision support is key to the high quality of nursing care for patients with complex conditions. Research in nursing pays little attention to CDSSs' ability to synthesize multiple guidelines to provide optimized and conflict-free decision support for nursing care of patients with comorbidity. The proposed conceptual framework addresses this gap and guides the development of a CDSS to support complex nursing care through the delivery of user-preferred and conflict-free decision support.

\section{Protection of Human and Animal Subjects}

This study was deemed exempt by the University Institutional Review Board.

\section{Authors' Contribution}

M.M. and S.-C.L. contributed toward conception and design; M.M. provided financial support. All the authors collected and analyzed the data, drafted and approved the manuscript.

\section{Funding}

The authors also acknowledge the financial support from the Office of the Vice President for Research, University of
Minnesota Grant-in-Aid. The content is solely the responsibility of the authors and does not necessarily represent the official views of the funding agency.

\section{Conflict of Interest}

None declared.

\section{Acknowledgments}

The authors thank the University of Minnesota, School of Nursing for their support.

\section{References}

1 Kawamoto K, Houlihan CA, Balas EA, Lobach DF. Improving clinical practice using clinical decision support systems: a systematic review of trials to identify features critical to success. BMJ 2005; 330(7494):765-768

2 Fillmore CL, Bray BE, Kawamoto K. Systematic review of clinical decision support interventions with potential for inpatient cost reduction. BMC Med Inform Decis Mak 2013; 13(01):135

3 Goldzweig CL, Orshansky G, Paige NM, et al. Electronic health record-based interventions for improving appropriate diagnostic imaging: a systematic review and meta-analysis. Ann Intern Med 2015;162(08):557-565

4 Lee $\mathrm{S}$. Features of computerized clinical decision support systems supportive of nursing practice: a literature review. Comput Inform Nurs 2013;31(10):477-495

5 Nibbelink CW, Carrington JM. Nurse decision making in acute care. Comput Inform Nurs 2019;37(03):151-160

6 Nibbelink CW, Brewer BB. Decision-making in nursing practice: an integrative literature review. J Clin Nurs 2018;27(5-6):917-928

7 Oliveira EM, Secco LMD, Figueiredo WB, Padilha KG, Secoli SR. Nursing Activities Score and the cost of nursing care required and available. Rev Bras Enferm 2019;72(Suppl 1):137-142

8 Welton JM, Harper EM. Measuring nursing care value. Nurs Econ 2016;34(01):7-14

9 Dunn Lopez K, Gephart SM, Raszewski R, Sousa V, Shehorn LE, Abraham J. Integrative review of clinical decision support for registered nurses in acute care settings. J Am Med Inform Assoc 2017;24(02):441-450

10 Borum C. Barriers for hospital-based nurse practitioners utilizing clinical decision support systems: a systematic review. Comput Inform Nurs 2018;36(04):177-182

11 Toma SDE, Para DED, Revisión E. Decision-making system for nursing: integrative review. J Nurs UFPE Line 2017;11 (Suppl 10):4263-4272

12 Khong PCB, Holroyd E, Wang W. A critical review of the theoretical frameworks and the conceptual factors in the adoption of clinical decision support systems. Comput Inform Nurs 2015;33(12): 555-570

13 Greenhalgh T, Wherton J, Papoutsi C, et al. Beyond adoption: a new framework for theorizing and evaluating nonadoption, abandonment, and challenges to the scale-up, spread, and sustainability of health and care technologies. J Med Internet Res 2017;19(11):e367

14 Banning M. A review of clinical decision making: models and current research. J Clin Nurs 2008;17(02):187-195

15 Bakken S, Currie LM, Lee N-J, Roberts WD, Collins SA, Cimino JJ. Integrating evidence into clinical information systems for nursing decision support. Int J Med Inform 2008;77(06): 413-420

16 Pelaccia T, Tardif J, Triby E, Charlin B. An analysis of clinical reasoning through a recent and comprehensive approach: the dual-process theory. Med Educ Online 2011;16(01). Doi: 10.3402/ meo.v16i0.5890 
17 Paley J, Cheyne H, Dalgleish L, Duncan EAS, Niven CA. Nursing's ways of knowing and dual process theories of cognition. J Adv Nurs 2007;60(06):692-701

18 Bjørk IT, Hamilton GA. Clinical decision making of nurses working in hospital settings. Nurs Res Pract 2011;2011:524918

19 Carper B. Fundamental patterns of knowing in nursing. ANS Adv Nurs Sci 1978;1(01):13-23

20 Price A, Zulkosky K, White K, Pretz J. Accuracy of intuition in clinical decision-making among novice clinicians. J Adv Nurs 2017;73(05):1147-1157

21 Hicks EP, Kluemper GT. Heuristic reasoning and cognitive biases: are they hindrances to judgments and decision making in orthodontics? Am J Orthod Dentofacial Orthop 2011;139(03):297-304

22 Featherston R, Downie LE, Vogel AP, Galvin KL. Decision making biases in the allied health professions: a systematic scoping review. PLoS One 2020;15(10):e0240716

23 Thompson C, Aitken L, Doran D, Dowding D. An agenda for clinical decision making and judgement in nursing research and education. Int J Nurs Stud 2013;50(12):1720-1726

24 Thompson C. A conceptual treadmill: the need for 'middle ground' in clinical decision making theory in nursing. J Adv Nurs 1999;30 (05):1222-1229

25 Pretz JE, Folse VN. Nursing experience and preference for intuition in decision making. J Clin Nurs 2011;20(19-20):2878-2889

26 Chilcote DR. Intuition: a concept analysis. Nurs Forum 2017;52 (01):62-67

27 Kawamoto K, Del Fiol G. Clinical decision support systems in healthcare. In: Nelson R, Staggers N, eds. Health Informatics: An Interprofessional Approach. 2nd ed. Elsevier; 2016:170-183

28 Osheroff JA, Teich JM, Levick D, et al. Improving Outcomes with Clinical Decision Support: An Implementer's Guide. 2nd ed. HIMSS; 2012

29 Clarke MA, Belden JL, Koopman RJ, et al. Information needs and information-seeking behaviour analysis of primary care physicians and nurses: a literature review. Health Info Libr J 2013;30 (03):178-190

30 Kilsdonk E, Peute LW, Jaspers MWM. Factors influencing implementation success of guideline-based clinical decision support systems: a systematic review and gaps analysis. Int J Med Inform 2017;98:56-64

31 Farokhzadian J, Khajouei R, Ahmadian L. Information seeking and retrieval skills of nurses: nurses readiness for evidence based practice in hospitals of a medical university in Iran. Int J Med Inform 2015;84(08):570-577

32 O'leary DF, Mhaolrúnaigh SN. Information-seeking behaviour of nurses: where is information sought and what processes are followed? J Adv Nurs 2012;68(02):379-390

33 Camargo FC, Iwamoto HH, Galvão CM, Pereira GA, Andrade RB, Masso GC. Competences and barriers for the evidence-based practice in nursing: an integrative review. Rev Bras Enferm 2018;71(04):2030-2038

34 Han Y, Zeng H, Jiang H, et al. CSC expert consensus on principles of clinical management of patients with severe emergent cardiovascular diseases during the COVID-19 epidemic. Circulation 2020;141(20):e810-e816

35 Alford D, Alexander S, Barr R. Optimization of clinical decision support tools for the care of older adults with diabetes mellitus type 2. Comput Inform Nurs 2018;36(06):259-264

36 Jeffery AD, Novak LL, Kennedy B, Dietrich MS, Mion LC. Participatory design of probability-based decision support tools for inhospital nurses. J Am Med Inform Assoc 2017;24(06):1102-1110

37 Brunner J, Chuang E, Goldzweig C, Cain CL, Sugar C, Yano EM. Usercentered design to improve clinical decision support in primary care. Int J Med Inform 2017;104(March):56-64

38 Cho I, Staggers N, Park I. Nurses' responses to differing amounts and information content in a diagnostic computer-based decision support application. Comput Inform Nurs 2010;28(02):95-102
39 Cvach M. Monitor alarm fatigue: an integrative review. Biomed Instrum Technol 2012;46(04):268-277

40 Greenes RA, Bates DW, Kawamoto K, Middleton B, Osheroff J, Shahar Y. Clinical decision support models and frameworks: Seeking to address research issues underlying implementation successes and failures. J Biomed Inform 2018;78(78):134-143

41 Freimuth RR, Formea CM, Hoffman JM, Matey E, Peterson JF, Boyce RD. Implementing genomic clinical decision support for drugbased precision medicine. CPT Pharmacometrics Syst Pharmacol 2017;6(03):153-155

42 World Health Organization. Multimorbidity: Technical Series on Safer Primary Care. 2016 Accessed October 30, 2019 at: http:// apps.who.int/bookorders

43 Fiset VJ, Graham ID, Davies BL. Evidence-based practice in clinical nursing education: a scoping review. J Nurs Educ 2017;56(09): 534-541

44 Carey S, Hogan S. Failure in systems and culture: barriers that prevent implementation of evidence-based fasting times for patients in the acute care setting. J Parenter Enter Nutr 2021;45 (05):933-940

45 Michalowski M, Wilk S, Michalowski W, Carrier M. Mitplan: a planning approach to mitigating concurrently applied clinical practice guidelines. In: Riaño D, Wilk S, ten Teije A, eds. Artificial Intelligence in Medicine. AIME 2019. Lecture Notes in Computer Science. Vol. 11526. Springer; 2019:93-103

46 Wilk S, Michalowski M, Michalowski W, Rosu D, Carrier M, Kezadri-Hamiaz M. Comprehensive mitigation framework for concurrent application of multiple clinical practice guidelines. J Biomed Inform 2017;66:52-71

47 Prentzas J, Hatzilygeroudis I. Categorizing approaches combining rule-based and case-based reasoning. Expert Syst 2007;24(02): 97-122

48 Yu PP. Knowledge bases, clinical decision support systems, and rapid learning in oncology. J Oncol Pract 2015;11(02):e206-e211

49 Battisto D, Pak R, Vander Wood MA, Pilcher JJ. Using a task analysis to describe nursing work in acute care patient environments. J Nurs Adm 2009;39(12):537-547

50 Douglas S, Cartmill R, Brown R, et al. The work of adult and pediatric intensive care unit nurses. Nurs Res 2013;62(01):50-58

51 Whelton PK, Carey RM, Aronow WS, et al. 2017 ACC/AHA/AAPA/ ABC/ACPM/AGS/APhA/ ASH/ASPC/NMA/PCNA guideline for the prevention, detection, evaluation, and management of high blood pressure in adults a report of the American College of Cardiology/American Heart Association Task Force on clinical pr. Hypertension 2018;71(06):e13-e115

52 Tsutsui $\mathrm{H}$, Isobe M, Ito H, et al; Japanese Circulation Society and the Japanese Heart Failure Society Joint Working Group. JCS 2017/ JHFS 2017 guideline on diagnosis and treatment of acute and chronic heart failure. Circ J 2019;83(10):2084-2184

53 Munshi MN, Florez H, Huang ES, et al. Management of diabetes in long-term care and skilled nursing facilities: a position statement of the American diabetes association. Diabetes Care 2016;39(02): 308-318

54 Herdman TH, Kamitsuru S, eds. NANDA International Nursing Diagnoses: Definitions \& Classification 2018-2020. 7th ed. Noida: Thieme Medical Publishers; 2017

55 Brush JE Jr, Handberg EM, Biga C, et al. 2015 ACC health policy statement on cardiovascular team-based care and the role of advanced practice providers. J Am Coll Cardiol 2015;65(19): 2118-2136

56 Sutton RT, Pincock D, Baumgart DC, Sadowski DC, Fedorak RN Kroeker KI. An overview of clinical decision support systems: benefits, risks, and strategies for success. NPJ Digit Med 2020;3 (01):17

57 Westra BL, Christie B, Johnson SG, et al. Modeling flowsheet data to support secondary use. Comput Inform Nurs 2017;35(09): $452-458$ 
58 O'Neill ES, Dluhy NM, Chin E. Modeling novice clinical reasoning for a computerized decision support system. J Adv Nurs 2005;49(01):67-77

59 Tacia L, Biskupski K, Pheley A, Lehto RH. Identifying barriers to evidence-based practice adoption: a focus group study. Clin Nurs Stud 2015;3(02):90-96

60 Harrison RL, Lyerla F. Using nursing clinical decision support systems to achieve meaningful use. Comput Inform Nurs 2012; 30(07):380-385

61 Karlsson LO, Nilsson S, Bång M, Nilsson L, Charitakis E, Janzon M. A clinical decision support tool for improving adherence to guidelines on anticoagulant therapy in patients with atrial fibrillation at risk of stroke: a cluster-randomized trial in a Swedish primary care setting (the CDS-AF study). PLoS Med 2018;15(03):e1002528

62 Stojkovska LA, Loskovska S. Clinical decision support systems: medical knowledge acquisition and representation methods. Paper presented at: 2010 IEEE Int Conf Electro/Information Technol EIT2010. Published online 2010:0-5
63 Vernooij RWM, Sanabria AJ, Solà I, Alonso-Coello P, Martínez García L. Guidance for updating clinical practice guidelines: a systematic review of methodological handbooks. Implement Sci 2014;9(01):3

64 Castillo RS, Kelemen A. Considerations for a successful clinical decision support system. Comput Inform Nurs 2013;31(07): 319-326

65 Cresswell KM, Lee L, Mozaffar H, Williams R, Sheikh ANIHR ePrescribing Programme Team. Sustained user engagement in health information technology: the long road from implementation to system optimization of computerized physician order entry and clinical decision support systems for prescribing in hospitals in England. Health Serv Res 2017;52(05): 1928-1957

66 Brunner MC, Sheehan SE, Yanke EM, et al. Joint design with providers of clinical decision support for value-based advanced shoulder imaging. Appl Clin Inform 2020;11(01):142-152 